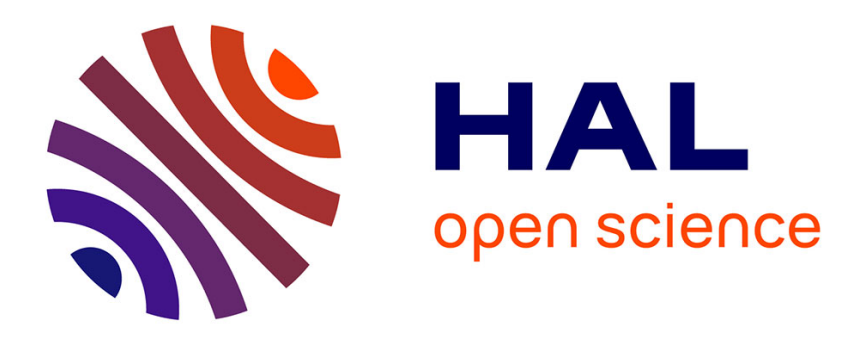

\title{
A slow transient diffusion in a drifted stable potential Arvind Singh
}

\section{To cite this version:}

Arvind Singh. A slow transient diffusion in a drifted stable potential. 2006. hal-00119374

\section{HAL Id: hal-00119374 https://hal.science/hal-00119374}

Preprint submitted on 8 Dec 2006

HAL is a multi-disciplinary open access archive for the deposit and dissemination of scientific research documents, whether they are published or not. The documents may come from teaching and research institutions in France or abroad, or from public or private research centers.
L'archive ouverte pluridisciplinaire HAL, est destinée au dépôt et à la diffusion de documents scientifiques de niveau recherche, publiés ou non, émanant des établissements d'enseignement et de recherche français ou étrangers, des laboratoires publics ou privés. 


\title{
A slow transient diffusion in a drifted stable potential
}

\author{
ARVind Singh * \\ Université Paris VI
}

\begin{abstract}
We consider a diffusion process $X$ in a random potential $\mathbb{V}$ of the form $\mathbb{V}_{x}=\mathbb{S}_{x}-\delta x$ where $\delta$ is a positive drift and $\mathbb{S}$ is a strictly stable process of index $\alpha \in(1,2)$ with positive jumps. Then the diffusion is transient and $X_{t} / \log ^{\alpha} t$ converges in law towards an exponential distribution. This behaviour contrasts with the case where $\mathbb{V}$ is a drifted Brownian motion and provides an example of a transient diffusion in a random potential which is as "slow" as in the recurrent setting.
\end{abstract}

Keywords. diffusion with random potential, stable processes

MSC 2000. 60K37, 60J60, 60F05

e-mail. arvind.singh@ens.fr

\section{Introduction}

Let $(\mathbb{V}(x), x \in \mathbb{R})$ be a two-sided stochastic process defined on some probability space $(\Omega, \mathcal{F}, \mathbf{P})$. We call a diffusion in the random potential $\mathbb{V}$ an informal solution $X$ of the S.D.E:

$$
\left\{\begin{array}{l}
d X_{t}=d \beta_{t}-\frac{1}{2} \mathbb{V}^{\prime}\left(X_{t}\right) d t \\
X_{0}=0
\end{array}\right.
$$

where $\beta$ is a standard Brownian motion independent of $\mathbb{V}$. Of course, the process $\mathbb{V}$ may not be differentiable (for example when $\mathbb{V}$ is a Brownian motion) and we should formally consider $X$ as a diffusion whose conditional generator given $\mathbb{V}$ is

$$
\frac{1}{2} e^{\mathbb{V}(x)} \frac{d}{d x}\left(e^{-\mathbb{V}(x)} \frac{d}{d x}\right) .
$$

Such a diffusion may be explicitly constructed from a Brownian motion through a random change of time and a random change of scale. This class of processes has

* Laboratoire de Probabilités et Modèles Aléatoires, Université Pierre et Marie Curie, 175 rue du Chevaleret, 75013 Paris, France. 
been widely studied for the last twenty years and bears a close connection with the model of the random walk in random environment (RWRE), see [17] and [12] for a survey on RWRE and [11], 12 for the connection between the two models.

This model exhibits many interesting features. For instance, when the potential process $\mathbb{V}$ is a Brownian motion, the diffusion $X$ is recurrent and Brox [2] proved that $X_{t} / \log ^{2} t$ converges to a non-degenerate distribution. Thus, the diffusion is much "slower" than in the trivial case $\mathbb{V}=0$ (then $X$ is simply a Brownian motion).

We point out that Brox's theorem is the analogue of Sinai's famous theorem for RWRE [13] (see also [4] and [8]). Just as for the RWRE, this result is a consequence of a so-called "localization phenomena": the diffusion is trapped in some valleys of its potential $\mathbb{V}$. Brox's theorem may also be extended to a wider class of potentials. For instance, when $\mathbb{V}$ is a strictly stable process of index $\alpha \in(0,2]$, Schumacher [1] proved that

$$
\frac{X_{t}}{\log ^{\alpha} t} \underset{t \rightarrow \infty}{\stackrel{\text { law }}{\longrightarrow}} b_{\infty}
$$

where $b_{\infty}$ is a non-degenerate random variable, whose distribution depends on the parameters of the stable process $\mathbb{V}$.

There is also much interest concerning the behaviour of $X$ in the transient case. When the potential is a drifted Brownian motion i.e. $\mathbb{V}_{x}=\mathbb{B}_{x}-\frac{\kappa}{2} x$ where $\mathbb{B}$ is a two-sided Brownian motion and $\kappa>0$, then the associated diffusion $X$ is transient toward $+\infty$ and its rate of growth is polynomial and depends on $\kappa$. Precisely, Kawazu and Tanaka [7] proved that

- If $0<\kappa<1$, then $\frac{1}{t^{\kappa}} X_{t}$ converges in law towards a Mittag-Leffler distribution of index $\kappa$.

- If $\kappa=1$, then $\frac{\log t}{t} X_{t}$ converges in probability towards $\frac{1}{4}$.

- If $\kappa>1$, then $\frac{1}{t} X_{t}$ converges almost surely towards $\frac{\kappa-1}{4}$.

In particular, when $\kappa<1$, the rate of growth of $X$ is sub-linear. Refined results on the rates of convergence for this process were later obtained by Tanaka [16] and $\mathrm{Hu}$ et al. [6].

In fact, this behaviour is not specific to diffusions in a drifted Brownian potential. More generally, it is proved in [15] that if $\mathbb{V}$ is a two-sided Lévy process with no positive jumps and if there exists $\kappa>0$ such $\mathbf{E}\left[e^{\kappa \mathbb{V}_{1}}\right]=1$, then the rate of growth of $X_{t}$ is linear when $\kappa>1$ and of order $t^{\kappa}$ when $0<\kappa<1$ (see also [3] for a law of large numbers in a general Lévy potential). These results are the analogues of those previously obtained by Kesten et al. [9] for the discrete model of the RWRE.

In this paper, we study the asymptotic behaviour of a diffusion in a drifted stable potential. Precisely, let $\left(\mathbb{S}_{x}, x \in \mathbb{R}\right)$ denote a two-sided càdlàg stable process with index $\alpha \in(1,2)$. By two-sided, we mean that

(a) The process $\left(\mathbb{S}_{x}, x \geq 0\right)$ is strictly stable with index $\alpha \in(1,2)$, in particular $\mathbb{S}_{0}=0$.

(b) For all $x_{0} \in \mathbb{R}$, the process $\left(\mathbb{S}_{x+x_{0}}-\mathbb{S}_{x_{0}}, x \in \mathbb{R}\right)$ has the same law as $\mathbb{S}$. 
It is well known that the Lévy measure $\Pi$ of $\mathbb{S}$ has the form

$$
\Pi(d x)=\left(c^{+} \mathbf{1}_{\{x>0\}}+c^{-} \mathbf{1}_{\{x<0\}}\right) \frac{d x}{|x|^{\alpha+1}}
$$

where $c^{+}$and $c^{-}$are two non-negative constants such that $c^{+}+c^{-}>0$. In particular, the process $\left(\mathbb{S}_{x}, x \geq 0\right.$ ) has no positive jumps (resp. no negative jumps) if and only if $c^{+}=0$ (resp. $c^{-}=0$ ). Given $\delta>0$, we consider a diffusion $X$ in the random potential

$$
\mathbb{V}_{x}=\mathbb{S}_{x}-\delta x .
$$

Since the index $\alpha$ of the stable process $\mathbb{S}$ is larger than 1 , we have $\mathbf{E}\left[\mathbb{V}_{x}\right]=-\delta x$, and therefore

$$
\lim _{x \rightarrow+\infty} \mathbb{V}_{x}=-\infty \text { and } \lim _{x \rightarrow-\infty} \mathbb{V}_{x}=+\infty \quad \text { almost surely. }
$$

This implies that the random diffusion $X$ is transient toward $+\infty$. We already mentioned that, when $\mathbb{S}$ has no positive jumps (i.e. $\left.c^{+}=0\right)$, the rate of transience of $X$ is given in 15 and $X_{t}$ has a polynomial growth. Thus, we now assume that $\mathbb{S}$ possesses positive jumps.

Theorem 1. Assume that $c^{+}>0$, then

$$
\frac{X_{t}}{\log ^{\alpha} t} \underset{t \rightarrow \infty}{\stackrel{\text { law }}{\longrightarrow}} \mathcal{E}\left(\frac{c^{+}}{\alpha}\right)
$$

where $\mathcal{E}\left(c^{+} / \alpha\right)$ denotes an exponential law with parameter $c^{+} / \alpha$. This result also holds with $\sup _{s \leq t} X_{s}$ or $\inf _{s \geq t} X_{s}$ in place of $X_{t}$.

The asymptotic behaviour of $X$ is in this case very different from the one observed when $\mathbb{V}$ is a drifted Brownian motion. Here, the rate of growth is very slow: it is the same as in the recurrent setting. We also note that neither the rate of growth nor the limiting law depend on the value of the drift parameter $\delta$.

Theorem 11 has a simple heuristic explanation: the "localisation phenomena" for the diffusion $X$ tells us that the time needed to reach a positive level $x$ is approximatively exponentially proportional to the biggest ascending barrier of $\mathbb{V}$ on the interval $[0, x]$. In the case of a Brownian potential, or more generally a spectrally negative Lévy potential, the addition of a negative drift somehow "kills" the ascending barriers, thus accelerating the diffusion and leading to a polynomial rate of transience. However, in our setting, the biggest ascending barrier on $[0, x]$ of the stable process $\mathbb{S}$ is of the same order as its biggest jump on this interval. Since, the addition of a drift has no influence on the jumps of the potential process, the time needed to reach level $x$ still remains of the same order as in the recurrent case (i.e. when the drift is zero) and yields a logarithmic rate of transience. 


\section{Proof of the theorem.}

\subsection{Representation of $X$ and of its hitting times.}

In the remainder of this paper, we indifferently use the notation $\mathbb{V}_{x}$ or $\mathbb{V}(x)$. Let us first recall the classical representation of the diffusion $X$ in the random potential $\mathbb{V}$ from a Brownian motion through a random change of scale and a random change of time (see [2] or [12] for details). Let $\left(B_{t}, t \geq 0\right)$ denote a standard Brownian motion independent of $\mathbb{V}$ and let $\sigma$ stand for its hitting times:

$$
\sigma(x) \stackrel{\text { def }}{=} \inf \left(t \geq 0, B_{t}=x\right) .
$$

Define the scale function of the diffusion $X$,

$$
\mathbb{A}(x) \stackrel{\text { def }}{=} \int_{0}^{x} e^{\mathbb{V} y} d y \quad \text { for } x \in \mathbb{R} .
$$

Since $\lim _{x \rightarrow+\infty} \mathbb{V}_{x} / x=-\delta$ and $\lim _{x \rightarrow-\infty} \mathbb{V}_{x} / x=\delta$ almost surely, it is clear that

$$
\mathbb{A}(\infty)=\lim _{x \rightarrow+\infty} \mathbb{A}(x)<\infty \quad \text { and } \quad \lim _{x \rightarrow-\infty} \mathbb{A}(x)=-\infty \quad \text { almost surely. }
$$

Let $\mathbb{A}^{-1}:(-\infty, \mathbb{A}(\infty)) \mapsto \mathbb{R}$ denote the inverse of $\mathbb{A}$ and define

$$
\mathbb{T}(t) \stackrel{\text { def }}{=} \int_{0}^{t} e^{-2 \mathbb{V}\left(\mathbb{A}^{-1}\left(B_{s}\right)\right)} d s \quad \text { for } 0 \leq t<\sigma(\mathbb{A}(\infty)) .
$$

Similarly, let $\mathbb{T}^{-1}$ denote the inverse of $\mathbb{T}$. According to Brox [2] (see also [12]), the diffusion $X$ in the random potential $\mathbb{V}$ may be represented in the form

$$
X_{t}=\mathbb{A}^{-1}\left(B_{\mathbb{T}^{-1}(t)}\right) \text {. }
$$

It is now clear that, under our assumptions, the diffusion $X$ is transient toward $+\infty$. We will study $X$ via its hitting times $H$ defined by

$$
H(r) \stackrel{\text { def }}{=} \inf \left(t \geq 0, X_{t}=r\right) \quad \text { for } r \geq 0 .
$$

Let $(L(t, x), t \geq 0, x \in \mathbb{R})$ stand for the bi-continuous version of the local time process of $B$. In view of (包), we can write

$$
H(r)=\mathbb{T}(\sigma(\mathbb{A}(r)))=\int_{0}^{\sigma(\mathbb{A}(r))} e^{-2 \mathbb{V}\left(\mathbb{A}^{-1}\left(B_{s}\right)\right)} d s=\int_{-\infty}^{\mathbb{A}(r)} e^{-2 \mathbb{V}\left(\mathbb{A}^{-1}(x)\right)} L(\sigma(\mathbb{A}(r)), x) d x .
$$

Making use of the change of variable $x=\mathbb{A}(y)$, we get

$$
H(r)=\int_{-\infty}^{r} e^{-\mathbb{V}_{y}} L(\sigma(\mathbb{A}(r)), \mathbb{A}(y)) d y=I_{1}(r)+I_{2}(r)
$$

where

$$
\begin{aligned}
& I_{1}(r) \stackrel{\text { def }}{=} \int_{0}^{r} e^{-\mathbb{V}_{y}} L(\sigma(\mathbb{A}(r)), \mathbb{A}(y)) d y \\
& I_{2}(r) \stackrel{\text { def }}{=} \int_{-\infty}^{0} e^{-\mathbb{V}_{y}} L(\sigma(\mathbb{A}(r)), \mathbb{A}(y)) d y .
\end{aligned}
$$




\subsection{Proof of Theorem 1 .}

Given a càdlàg process $\left(Z_{t}, t \geq 0\right)$, we denote by $\Delta_{t} Z=Z_{t}-Z_{t-}$ the size of the jump at time $t$. We also use the notation $Z_{t}^{\natural}$ to denote the largest positive jump of $Z$ before time $t$,

$$
Z_{t}^{\natural} \stackrel{\text { def }}{=} \sup \left(\Delta_{s}, 0 \leq s \leq t\right) .
$$

Let $Z_{t}^{\#}$ stand for the largest ascending barrier before time $t$, namely:

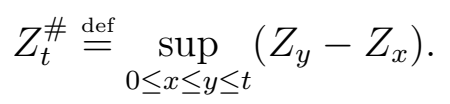

We also define the functionals:

$$
\begin{array}{ll}
\bar{Z}_{t} \stackrel{\text { def }}{=} \sup _{s \in[0, t]} Z_{s} & \text { (running unilateral maximum) } \\
\underline{Z}_{t} \stackrel{\text { def }}{=} \inf _{s \in[0, t]} Z_{s} & \text { (running unilateral minimum) } \\
Z_{t}^{*} \stackrel{\text { def }}{=} \sup _{s \in[0, t]}\left|Z_{s}\right| & \text { (running bilateral supremum) }
\end{array}
$$

We start with a simple lemma concerning the fluctuations of the potential process.

Lemma 1. There exist two constants $c_{1}, c_{2}>0$ such that for all $a, x>0$

$$
\mathbf{P}\left\{\mathbb{V}_{x}^{\#} \leq a\right\} \leq e^{-c_{1} \frac{x}{a^{\alpha}}}
$$

and whenever $\frac{a}{x}$ is sufficiently large,

$$
\mathbf{P}\left\{\mathbb{V}_{x}^{*}>a\right\} \leq c_{2} \frac{x}{a^{\alpha}}
$$

Proof. Recall that $\mathbb{V}_{x}=\mathbb{S}_{x}-\delta x$. In view of the form of the density of the Lévy measure of $\mathbb{S}$ given in (1), we get

$$
\mathbf{P}\left\{\mathbb{V}_{x}^{\#} \leq a\right\} \leq \mathbf{P}\left\{\mathbb{V}_{x}^{\natural} \leq a\right\}=\exp \left(-x \int_{a}^{\infty} \frac{c^{+}}{y^{\alpha}} d y\right)=\exp \left(-\frac{c^{+}}{\alpha} \frac{x}{a^{\alpha}}\right) .
$$

This yields (5). From the scaling property of the stable process $\mathbb{S}$, we also have

$$
\mathbf{P}\left\{\mathbb{V}_{x}^{*}>a\right\}=\mathbf{P}\left\{x^{\frac{1}{\alpha}} \sup _{t \in[0,1]}\left|\mathbb{S}_{t}-\delta x^{1-\frac{1}{\alpha}} t\right|>a\right\} \leq \mathbf{P}\left\{\mathbb{S}_{1}^{*}>\frac{a}{x^{\frac{1}{\alpha}}}-\delta x^{1-\frac{1}{\alpha}}\right\}
$$

Notice further that $a / x^{1 / \alpha}-\delta x^{1-1 / \alpha}>a /\left(2 x^{1 / \alpha}\right)$ whenever $a / x$ is large enough, therefore, making use of a classical estimate concerning the tail distribution of the stable process $\mathbb{S}$ (c.f. Proposition 4, p221 of [1]), we find that

$$
\mathbf{P}\left\{\mathbb{V}_{x}^{*}>a\right\} \leq \mathbf{P}\left\{\mathbb{S}_{1}^{*}>\frac{a}{2 x^{\frac{1}{\alpha}}}\right\} \leq \mathbf{P}\left\{\overline{\mathbb{S}}_{1}>\frac{a}{2 x^{\frac{1}{\alpha}}}\right\}+\mathbf{P}\left\{\mathbb{S}_{1}>\frac{a}{2 x^{\frac{1}{\alpha}}}\right\} \leq c_{2} \frac{x}{a^{\alpha}}
$$


Proposition 1. There exists a constant $c_{3}>0$ such that, for all $r$ sufficiently large and all $x \geq 0$,

$\mathbf{P}\left\{\mathbb{V}_{r}^{\#} \geq x+\log ^{4} r\right\}-c_{3} e^{-\log ^{2} r} \leq \mathbf{P}\left\{\log I_{1}(r) \geq x\right\} \leq \mathbf{P}\left\{\mathbb{V}_{r}^{\#} \geq x-\log ^{4} r\right\}+c_{3} e^{-\log ^{2} r}$.

Proof. This estimate was first proved by Hu and Shi (see Lemma 4.1 of [5]) when the potential process is close to a standard Brownian motion. A similar result is given in Proposition 3.2 of 14 when $\mathbb{V}$ is a random walk in the domain of attraction of a stable law. As explained by Shi [12], the key idea is the combined use of Ray-Knight's Theorem and Laplace's method. However, in our setting, additional difficulties appear since the potential process is neither flat on integer interval nor continuous. We shall therefore give a complete proof but one can still look in [5] and |14 for additional details. Recall that

$$
I_{1}(r)=\int_{0}^{r} e^{-\mathbb{V}_{y}} L(\sigma(\mathbb{A}(r)), \mathbb{A}(y)) d y,
$$

where $L$ is the local time of the Brownian motion $B$ (independent of $\mathbb{V}$ ). Let $(U(t), t \geq 0)$ denote a two-dimensional squared Bessel process starting from zero, also independent of $\mathbb{V}$. According to the first Ray-Knight Theorem (c.f. Theorem $2.2 \mathrm{p} 455$ of [10]), for any $x>0$ the process $(L(\sigma(x), x-y), 0 \leq y \leq x)$ has the same law as $(U(y), 0 \leq y \leq x)$. Therefore, making use of the scaling property of the Brownian motion and the independence of $\mathbb{V}$ and $B$, for each fixed $r>0$, the random variable $I_{1}(r)$ has the same law as

$$
\widetilde{I}_{1}(r) \stackrel{\text { def }}{=} \mathbb{A}(r) \int_{0}^{r} e^{-\mathbb{V}_{y}} U\left(\frac{\mathbb{A}(r)-\mathbb{A}(y)}{\mathbb{A}(r)}\right) d y .
$$

We simply need to prove the proposition for $\widetilde{I}_{1}$ instead of $I_{1}$. In the rest of the proof, we assume that $r$ is very large. We start with the upper bound. Define the event

$$
\mathcal{E}_{1} \stackrel{\text { def }}{=}\left\{\sup _{t \in(0,1]} \frac{U(t)}{t \log \left(\frac{8}{t}\right)} \leq r\right\} .
$$

According to Lemma 6.1 of [5], $\mathbf{P}\left\{\mathcal{E}_{1}^{c}\right\} \leq c_{4} e^{-r / 2}$ for some constant $c_{4}>0$. On $\mathcal{E}_{1}$, we have

$$
\begin{aligned}
\widetilde{I}_{1}(r) & \leq r \int_{0}^{r} e^{-\mathbb{V}_{y}}(\mathbb{A}(r)-\mathbb{A}(y)) \log \left(\frac{\mathbb{A}(r)}{\mathbb{A}(r)-\mathbb{A}(y)}\right) d y \\
& =r \int_{0}^{r}\left(\int_{y}^{r} e^{\mathbb{V}_{z}-\mathbb{V}_{y}} d z\right) \log \left(\frac{\mathbb{A}(r)}{\mathbb{A}(r)-\mathbb{A}(y)}\right) d y \\
& \leq r^{2} e^{\mathbb{V}_{r}^{\#}} \int_{0}^{r} \log \left(\frac{\mathbb{A}(r)}{\mathbb{A}(r)-\mathbb{A}(y)}\right) d y .
\end{aligned}
$$

Notice also that $\mathbb{A}(r)=\int_{0}^{r} e^{\mathbb{V}_{z}} d z \leq r e^{\overline{\mathbb{V}}_{r}}$ and similarly $\mathbb{A}(r)-\mathbb{A}(y) \geq(r-y) e^{\underline{\mathbb{V}}_{r}}$. Therefore

$$
\begin{aligned}
\int_{0}^{r} \log \left(\frac{\mathbb{A}(r)}{\mathbb{A}(r)-\mathbb{A}(y)}\right) d y & \leq r\left(\overline{\mathbb{V}}_{r}-\underline{\mathbb{V}}_{r}\right)+\int_{0}^{r} \log \left(\frac{8 r}{r-y}\right) d y \\
& =r\left(\overline{\mathbb{V}}_{r}-\underline{\mathbb{V}}_{r}+1+\log 8\right)
\end{aligned}
$$


Define the set $\mathcal{E}_{2} \stackrel{\text { def }}{=}\left\{\overline{\mathbb{V}}_{r}-\underline{\mathbb{V}}_{r} \leq e^{\log ^{3} r}\right\}$. In view of Lemma $\mathbb{1}$,

$$
\mathbf{P}\left\{\mathcal{E}_{2}^{c}\right\} \leq \mathbf{P}\left\{\mathbb{V}_{r}^{*}>\frac{1}{2} e^{\log ^{3} r}\right\} \leq e^{-\log ^{2} r} .
$$

Therefore, $\mathbf{P}\left\{\left(\mathcal{E}_{1} \cap \mathcal{E}_{2}\right)^{c}\right\} \leq 2 e^{-\log ^{2} r}$ and on $\mathcal{E}_{1} \cap \mathcal{E}_{2}$,

$$
\widetilde{I}_{1}(r) \leq r^{3}\left(e^{\log ^{3} r}+1+\log 8\right) e^{\mathbb{V}_{r}^{\#}} \leq e^{\log ^{4} r+\mathbb{V}_{r}^{\#}} .
$$

This completes the proof of the upper bound. We now deal with the lower bound. Define the sequence $\left(\gamma_{k}, k \geq 0\right)$ by induction

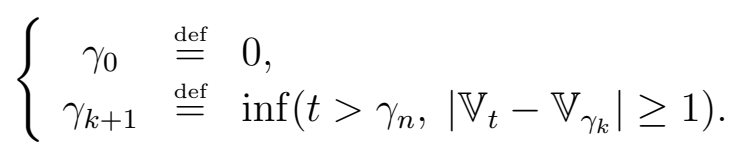

The sequence $\left(\gamma_{k+1}-\gamma_{k}, k \geq 0\right)$ is i.i.d. and distributed as $\gamma_{1}=\inf \left(t>0,\left|\mathbb{V}_{t}\right| \geq 1\right)$. We denote by $[x]$ the integer part of $x$. We also use the notation $\epsilon \stackrel{\text { def }}{=} e^{-\log ^{3} r}$. Consider the following events

$$
\begin{aligned}
& \mathcal{E}_{3} \stackrel{\text { def }}{=}\left\{\gamma_{\left[r^{2}\right]}>r\right\}, \\
& \mathcal{E}_{4} \stackrel{\text { def }}{=}\left\{\gamma_{k}-\gamma_{k-1} \geq 2 \epsilon \text { for all } k=1,2 \ldots,\left[r^{2}\right]\right\} .
\end{aligned}
$$

In view of Cramer's large deviation Theorem and since $r$ is very large, we get that $\mathbf{P}\left\{\mathcal{E}_{3}^{c}\right\} \leq e^{-r}$. We also have

$$
\begin{aligned}
\mathbf{P}\left\{\mathcal{E}_{4}^{c}\right\} \leq \sum_{k=1}^{\left[r^{2}\right]} \mathbf{P}\left\{\gamma_{k}-\gamma_{k-1}<2 \epsilon\right\} & \leq\left[r^{2}\right] \mathbf{P}\left\{\gamma_{1}<2 \epsilon\right\} \\
& \leq\left[r^{2}\right] \mathbf{P}\left\{\mathbb{V}_{2 \epsilon}^{*} \geq 1\right\} \\
& \leq e^{-\log ^{2} r}
\end{aligned}
$$

where we used Lemma 1 for the last inequality. Define also

$$
\mathcal{E}_{5} \stackrel{\text { def }}{=}\left\{\left|\mathbb{V}_{x}-\mathbb{V}_{r}\right|<1 \text { for all } x \in[r-2 \epsilon, r]\right\} .
$$

From time reversal, the processes $\left(\mathbb{V}_{t}, 0 \leq t \leq 2 \epsilon\right)$ and $\left(\mathbb{V}_{r}-\mathbb{V}_{(r-t)^{-}}, 0 \leq t \leq 2 \epsilon\right)$ have the same law. Thus,

$$
\mathbf{P}\left\{\mathcal{E}_{5}^{c}\right\} \leq \mathbf{P}\left\{\mathbb{V}_{2 \epsilon}^{*} \geq 1\right\} \leq e^{-\log ^{2} r} .
$$

Setting $\mathcal{E}_{6} \stackrel{\text { def }}{=} \mathcal{E}_{3} \cap \mathcal{E}_{4} \cap \mathcal{E}_{5}$, we get $\mathbf{P}\left\{\mathcal{E}_{6}^{c}\right\} \leq 3 e^{-\log ^{2} r}$. Moreover, it is easy to check (see figure 1 ) that on $\mathcal{E}_{6}$, we can always find $x_{-}, x_{+}$such that:

$$
\left\{\begin{array}{l}
0 \leq x_{-} \leq x_{+} \leq r-2 \epsilon \\
\text { for any } a \in\left[x_{-}, x_{-}+\epsilon\right],\left|\mathbb{V}_{x_{-}}-\mathbb{V}_{a}\right| \leq 2, \\
\text { for any } b \in\left[x_{+}, x_{+}+\epsilon\right],\left|\mathbb{V}_{x_{+}}-\mathbb{V}_{b}\right| \leq 2 \\
\mathbb{V}_{x_{+}}-\mathbb{V}_{x_{-}} \geq \mathbb{V}_{r}^{\#}-4
\end{array}\right.
$$




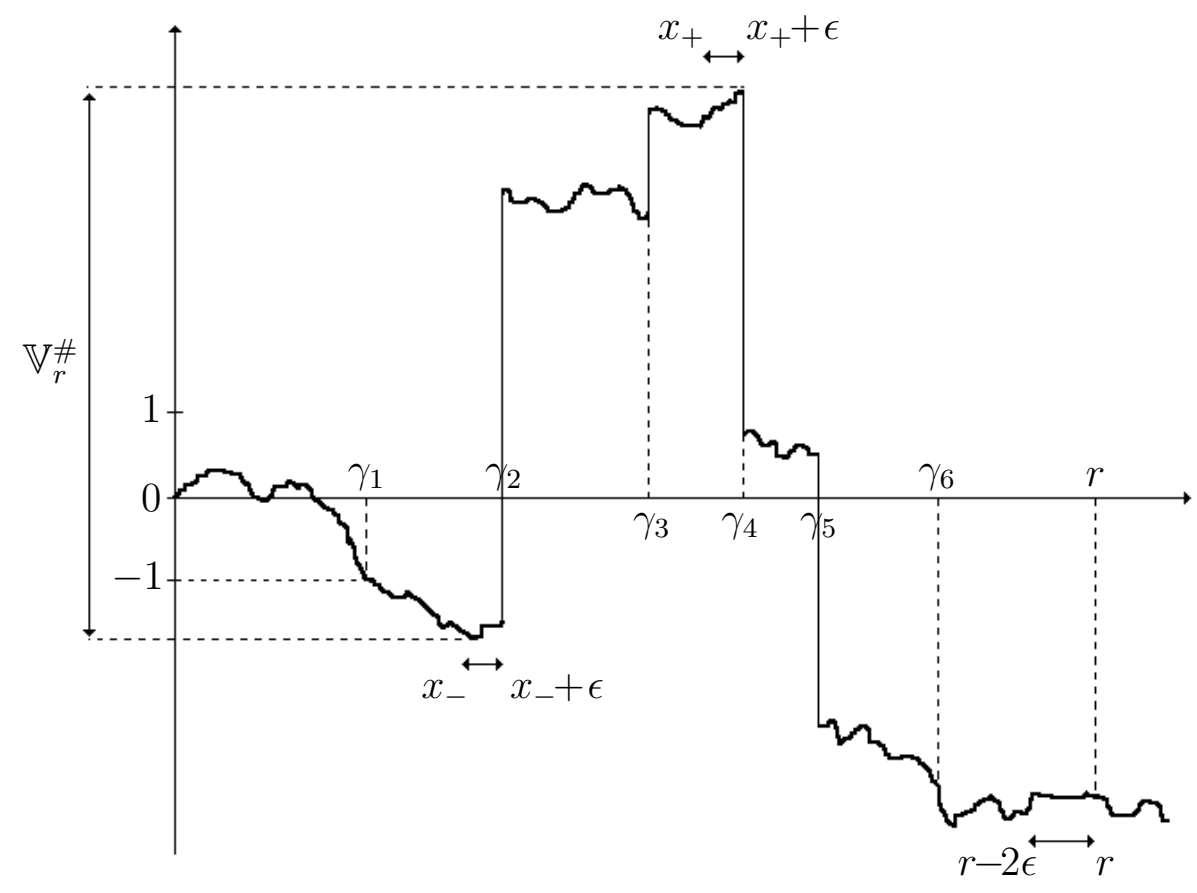

Figure 1: Sample path of $\mathbb{V}$ on $\mathcal{E}_{6}$.

Let us also define

$$
\begin{aligned}
& \mathcal{E}_{7} \stackrel{\text { def }}{=} \mathcal{E}_{6} \cap\left\{\inf _{y \in\left[x_{-}, x_{-}+\epsilon\right]} U\left(\frac{\mathbb{A}(r)-\mathbb{A}(y)}{\mathbb{A}(r)}\right) \geq \frac{\mathbb{A}(r)-\mathbb{A}\left(x_{-}\right)}{\mathbb{A}(r)} e^{-2 \log ^{2} r}\right\}, \\
& \mathcal{E}_{8} \stackrel{\text { def }}{=}\left\{\mathbb{V}_{r}^{\natural} \geq 3 \log ^{2} r\right\} .
\end{aligned}
$$

We finally set $\mathcal{E}_{9} \stackrel{\text { def }}{=} \mathcal{E}_{7} \cap \mathcal{E}_{8}$. Then on $\mathcal{E}_{9}$, we have, for all $r$ large enough,

$$
\begin{aligned}
\widetilde{I}_{1}(r) & \geq \mathbb{A}(r) \int_{x_{-}}^{x_{-}+\epsilon} e^{-\mathbb{V}_{y}} U\left(\frac{\mathbb{A}(r)-\mathbb{A}(y)}{\mathbb{A}(r)}\right) d y \\
& \geq e^{-\mathbb{V}_{x_{-}-2-2 \log ^{2} r}} \int_{x_{-}}^{x_{-}+\epsilon}\left(\mathbb{A}(r)-\mathbb{A}\left(x_{-}\right)\right) d y \\
& =e^{-\mathbb{V}_{x_{-}-2-2 \log ^{2} r-\log ^{3} r}} \int_{x_{-}}^{r} e^{\mathbb{V}_{y}} d y \\
& \geq e^{-\mathbb{V}_{x_{-}-2-2 \log ^{2} r-\log ^{3} r}} \int_{x_{+}}^{x_{+}+\epsilon} e^{\mathbb{V}_{y}} d y \\
& \geq e^{\mathbb{V}_{x_{+}-\mathbb{V}_{x_{-}}-4-2 \log ^{2} r-2 \log ^{3} r}} \\
& \geq e^{\mathbb{V}_{r}^{\#}-\log ^{4} r} .
\end{aligned}
$$

This proves the lower bound on $\mathcal{E}_{9}$. It simply remains to show that $\mathbf{P}\left\{\mathcal{E}_{9}^{c}\right\} \leq$ 
$c_{5} e^{-\log ^{2} r}$. According to Lemma 6.1 of [5], for any $0<a<b$ and any $\eta>0$, we have

$$
\mathbf{P}\left\{\inf _{a<t<b} U(t) \leq \eta b\right\} \leq 2 \sqrt{\eta}+2 \exp \left(-\frac{\eta}{2(1-a / b)}\right) .
$$

Therefore, making use of the independence of $\mathbb{V}$ and $U$, we find

$$
\begin{aligned}
\mathbf{P}\left\{\mathcal{E}_{9}^{c}\right\} & \leq \mathbf{P}\left\{\mathcal{E}_{6}^{c}\right\}+\mathbf{P}\left\{\mathcal{E}_{8}^{c}\right\}+\mathbf{P}\left\{\mathcal{E}_{7}^{c} \cap \mathcal{E}_{6} \cap \mathcal{E}_{8}\right\} \\
& \leq \mathbf{P}\left\{\mathcal{E}_{6}^{c}\right\}+\mathbf{P}\left\{\mathcal{E}_{8}^{c}\right\}+2 e^{-\log ^{2} r}+2 \mathbf{E}\left[e^{\frac{1}{2} \mathbb{J}(r) e^{-2 \log ^{2} r}} \mathbf{1}_{\mathcal{E}_{6} \cap \mathcal{E}_{8}}\right]
\end{aligned}
$$

where

$$
\mathbb{J}(r) \stackrel{\text { def }}{=} \frac{\mathbb{A}(r)-\mathbb{A}\left(x_{-}\right)}{\mathbb{A}\left(x_{-}+\epsilon\right)-\mathbb{A}\left(x_{-}\right)} .
$$

We have already proved that $\mathbf{P}\left\{\mathcal{E}_{6}^{c}\right\} \leq 3 e^{-\log ^{2} r}$. Using Lemma 1, we also check that $\mathbf{P}\left\{\mathcal{E}_{8}^{c}\right\} \leq e^{-\log ^{2} r}$. Thus, it remains to show that

$$
\mathbf{E}\left[e^{\frac{1}{2} \mathbb{J}(r) e^{-2 \log ^{2} r}} \mathbf{1}_{\mathcal{E}_{6} \cap \mathcal{E}_{8}}\right] \leq c_{6} e^{-\log ^{2} r} .
$$

Notice that, on $\mathcal{E}_{6}$,

$$
\mathbb{A}(r)-\mathbb{A}\left(x_{-}\right)=\int_{x_{-}}^{r} e^{\mathbb{V}_{y}} d y \geq \int_{x_{+}}^{x_{+}+\epsilon} e^{\mathbb{V}_{y}} d y \geq e^{\log ^{3} r+\mathbb{V}_{x_{+}}-2},
$$

and also

$$
\mathbb{A}\left(x_{-}+\epsilon\right)-\mathbb{A}\left(x_{-}\right)=\int_{x_{-}}^{x_{-}+\epsilon} e^{\mathbb{V}_{y}} d y \leq e^{\log ^{3} r+\mathbb{V}_{x_{-}}+2} .
$$

Therefore, on $\mathcal{E}_{6} \cap \mathcal{E}_{8}$,

$$
\mathbb{J}(r) \geq e^{\mathbb{V}_{x_{+}}-\mathbb{V}_{x_{-}-4}} \geq e^{\mathbb{V}_{r}^{\#}-8} \geq e^{\mathbb{V}_{r}^{\natural}-8} \geq e^{3 \log ^{2} r-8}
$$

which clearly yields (7) and the proof of the proposition is complete.

Lemma 2. We have

$$
\frac{\mathbb{V}_{r}^{\#}}{r^{1 / \alpha}} \underset{r \rightarrow \infty}{\stackrel{\text { law }}{\longrightarrow}} \mathbb{S}_{1}^{\natural}
$$

Proof. Let $f:[0,1] \mapsto \mathbb{R}$ be a deterministic càdlàg function. For $\lambda \geq 0$, define

$$
f_{\lambda}(x) \stackrel{\text { def }}{=} f(x)-\lambda x .
$$

We first show that

$$
\lim _{\lambda \rightarrow \infty} f_{\lambda}^{\#}(1)=f^{\natural}(1) .
$$

It is clear that $f^{\natural}(1)=f_{\lambda}^{\natural}(1) \leq f_{\lambda}^{\#}(1)$ for any $\lambda>0$. Thus, we simply need to prove that $\lim \sup f_{\lambda}^{\#}(1) \leq f^{\natural}(1)$. Let $\eta>0$ and set

$$
\begin{aligned}
& A(\eta, \lambda) \stackrel{\text { def }}{=} \sup \left(f_{\lambda}(y)-f_{\lambda}(x), 0 \leq x \leq y \leq 1 \text { and } y-x \leq \eta\right), \\
& B(\eta, \lambda) \stackrel{\text { def }}{=} \sup \left(f_{\lambda}(y)-f_{\lambda}(x), 0 \leq x \leq y \leq 1 \text { and } y-x>\eta\right),
\end{aligned}
$$


so that

$$
f_{\lambda}^{\#}(1)=\max (A(\eta, \lambda), B(\eta, \lambda)) .
$$

Notice that $A(\eta, \lambda) \leq A(\eta)$ where

$$
A(\eta) \stackrel{\text { def }}{=} A(\eta, 0)=\sup (f(y)-f(x), 0 \leq x \leq y \leq 1 \text { and } y-x \leq \eta) .
$$

Since $f$ is càdlàg, we have $\lim _{\eta \rightarrow 0} A(\eta)=f^{\natural}(1)$. Thus, for any $\varepsilon>0$, we can find $\eta_{0}>0$ small enough such that

$$
\limsup _{\lambda \rightarrow \infty} A\left(\eta_{0}, \lambda\right) \leq f^{\natural}(1)+\varepsilon .
$$

Notice also that

$$
\begin{aligned}
B\left(\eta_{0}, \lambda\right) & \leq \sup \left(f(y)-f(x)-\eta_{0} \lambda, 0 \leq x \leq y \leq 1 \text { and } y-x>\eta_{0}\right) \\
& \leq f^{\#}(1)-\eta_{0} \lambda
\end{aligned}
$$

which implies

$$
\lim _{\lambda \rightarrow \infty} B\left(\eta_{0}, \lambda\right)=-\infty .
$$

The combination of (9), (10) and (11) yield (8). Making use of the scaling property of the stable process $\mathbb{S}$, for any fixed $r>0$,

$$
\left(\mathbb{V}_{y}, 0 \leq y \leq r\right) \stackrel{\text { law }}{=}\left(r^{1 / \alpha} \mathbb{S}_{y}-\delta r y, 0 \leq y \leq 1\right)
$$

Therefore, setting $\mathbb{R}(z)=(\mathbb{S} \text {. }-z \cdot)_{1}^{\#}$, we get the equality in law:

$$
\frac{\mathbb{V}_{r}^{\#}}{r^{1 / \alpha}} \stackrel{\text { law }}{=} \mathbb{R}\left(\delta r^{1-1 / \alpha}\right)
$$

Making use of (8), we see that $\mathbb{R}(z)$ converges almost surely towards $\mathbb{S}_{1}^{\natural}$ as $z$ goes to infinity. Since $\alpha>1$ and $\delta>0$, we also have $\delta r^{1-1 / \alpha} \rightarrow \infty$ as $r$ goes to infinity and we conclude from (12) that

$$
\frac{\mathbb{V}_{r}^{\#}}{r^{1 / \alpha}} \underset{r \rightarrow \infty}{\stackrel{\text { law }}{\longrightarrow}} \mathbb{S}_{1}^{\natural}
$$

Proof of Theorem 1 . Recall that the random variable $\mathbb{S}_{1}^{\natural}$ denotes the largest positive jump of $\mathbb{S}$ over the interval $[0,1]$. Thus, according to the density of the Lévy measure of $\mathbb{S}$,

$$
\mathbf{P}\left\{\mathbb{S}_{1}^{\natural} \leq x\right\}=\exp \left(-\int_{x}^{\infty} \frac{c^{+}}{y^{\alpha+1}} d y\right)=\exp \left(-\frac{c^{+}}{\alpha y^{\alpha}}\right) .
$$

On the one hand, the combination of Lemma 11 and 2 readily shows that

$$
\frac{\log \left(I_{1}(r)\right)}{r^{1 / \alpha}} \underset{r \rightarrow \infty}{\stackrel{\text { law }}{\longrightarrow}} \mathbb{S}_{1}^{\natural}
$$

On the other hand, the random variables $\mathbb{A}(\infty)=\lim _{x \rightarrow \infty} \mathbb{A}(x)$ and $\int_{-\infty}^{0} e^{-\mathbb{V}_{y}} d y$ have the same law. Moreover, we already noticed that these random variables are 
almost surely finite. Since the function $L(t, \cdot)$ is, for any fixed $t$, continuous with compact support, we get

$$
I_{2}(r)=\int_{-\infty}^{0} e^{-\mathbb{V}_{y}} L(\sigma(\mathbb{A}(r)), \mathbb{A}(y)) d y \leq \sup _{z \in(-\infty, 0]} L(\sigma(\mathbb{A}(\infty)), z) \int_{-\infty}^{0} e^{-\mathbb{V}_{y}} d y<\infty .
$$

Therefore,

$$
\sup _{r \geq 0} I_{2}(r)<\infty \quad \text { almost surely. }
$$

Combining (4), (14) and (15), we deduce that

$$
\frac{\log (H(r))}{r^{1 / \alpha}} \underset{r \rightarrow \infty}{\stackrel{\text { law }}{\longrightarrow}} \mathbb{S}_{1}^{\natural}
$$

which, from the definition of the hitting times $H$, yields

$$
\frac{\sup _{s \leq t} X_{s}}{\log ^{\alpha} t} \underset{t \rightarrow \infty}{\stackrel{\text { law }}{\longrightarrow}}\left(\frac{1}{\mathbb{S}_{1}^{\natural}}\right)^{\alpha} .
$$

According to $(13)$, the random variable $\left(1 / \mathbb{S}_{1}^{\natural}\right)^{\alpha}$ has an exponential distribution with parameter $c^{+} / \alpha$ so the proof of the theorem for $\sup _{s \leq t} X_{s}$ is complete. We finally use the classical argument given by Kawazu and Tanaka, p201 [7] to obtain the corresponding results for $X_{t}$ and $\inf _{s \geq t} X_{s}$.

Acknowledgments. I would like to thank Yueyun Hu for his precious advices.

\section{References}

[1] Bertoin, J. (1996). Lévy processes, Cambridge Univ. Press, Cambridge.

[2] Brox, Th. (1986). A one-dimensional diffusion process in a Wiener medium, Ann. Probab. 14, no. 4, 1206-1218.

[3] Carmona, P. (1997). The mean velocity of a Brownian motion in a random Lévy potential, Ann. Probab. 25, no. 4, 1774-1788.

[4] Golosov, A. O. (1986). Limit distributions for a random walk in a critical onedimensional random environment, Uspekhi Mat. Nauk 41, no. 2(248), 189-190.

[5] Hu, Y., ShI, Z. (1998). The limits of Sinai's simple random walk in random environment, Ann. Probab. 26, no. 4, 1477-1521.

[6] Hu, Y., ShI, Z. and Yor, M. (1999). Rates of convergence of diffusions with drifted Brownian potentials, Trans. Amer. Math. Soc. 351, no. 10, 3915-3934.

[7] Kawazu, K. and Tanaka, H. (1997). A diffusion process in a Brownian environment with drift, J. Math. Soc. Japan 49, no. 2, 189-211.

[8] Kesten, H. (1986). The limit distribution of Sinai's random walk in random environment, Phys. A 138, no. 1-2, 299-309. 
[9] Kesten, H., Kozlov, M. V. and Spitzer, F. (1975). A limit law for random walk in a random environment, Compositio Math. 30, 145-168.

[10] Revuz, D. and Yor, M. (1999). Continuous martingales and Brownian motion, Third edition, Springer, Berlin.

[11] Schumacher, S. (1985). Diffusions with random coefficients, in Particle systems, random media and large deviations (Brunswick, Maine, 1984), 351-356, Contemp. Math., 41, Amer. Math. Soc., Providence, RI.

[12] SHI, Z. (2001). Sinai's walk via stochastic calculus, in Milieux aléatoires, 53-74, Soc. Math. France, Paris.

[13] Sinai, YA. G. (1982). The limiting behavior of a one-dimensional random walk in a random environment, Teor. Veroyatnost. i Primenen. 27, no. 2, 247-258.

[14] Singh, A. (2007). Limiting behavior of a diffusion in an asymptotically stable environment, Ann. Inst. H. Poincaré Probab. Statist. 43, no. 1, 101-138

[15] Singh, A. (2007). Rates of convergence of a transient diffusion in a spectrally negative Lévy potential, submitted, available at http://arxiv.org/abs/math.PR/0606411

[16] TANakA, H. (1997). Limit theorems for a Brownian motion with drift in a white noise environment, Chaos Solitons Fractals 8, no. 11, 1807-1816.

[17] Zeitouni, O. (2004). Random walks in random environment, in Lectures on probability theory and statistics, 189-312, Lecture Notes in Math., 1837, Springer, Berlin. 